\title{
Double-Stranded RNA Dependent Kinase R Regulates Antibacterial Immunity in Sepsis
}

\author{
Yanliang Yang ${ }^{a}$ Lingli Xie $^{b, c}$ Yanjun Zhong ${ }^{d}$ Xiaoli Zhong ${ }^{b}$ Ran Meng ${ }^{b}$ \\ Qianqian Xue $^{\mathrm{b}}$ Fang Liang $^{\mathrm{b}}$ Kai Zhao $^{\mathrm{b}}$ Yiting Tang $^{\mathrm{a}}$ \\ aDepartment of Physiology, School of Basic Medical Science, Central South University, Changsha, PR China; \\ bDepartment of Hematology and Key Laboratory of Non-resolving Inflammation and Cancer of Hunan Province, \\ Central South University, Changsha, PR China; ' Department of Pathophysiology, Medical School, Hunan University \\ of Chinese Medicine, Changsha, PR China; ${ }^{\text {T} T h e ~ S e c o n d ~ X i a n g y a ~ H o s p i t a l, ~ C e n t r a l ~ S o u t h ~ U n i v e r s i t y, ~ C h a n g s h a, ~}$ \\ PR China
}

\section{Keywords}

Double-stranded RNA dependent kinase R - Bacterial sepsis . Interleukin-1

\section{Abstract}

Double-stranded RNA dependent kinase $R(P K R)$ is originally identified as an intracellular sensor of viral infection, but its role in bacterial infection remains largely unknown. Here we report that PKR was an important regulator of antibacterial immunity in sepsis. Genetic deletion of PKR or pharmacological inhibition of its kinase activity markedly increased bacterial loads, organ injury, and mortality in polymicrobial infection induced by cecal ligation and puncture (CLP). In contrast, PKR deficiency or inhibition did not affect bacterial loads, organ injury, or mortality when mice were systemically challenged with Escherichia coli, an abundant microbe in the gastrointestinal tract. PKR deficiency or inhibition markedly decreased the release of interleukin (IL)-1 $\beta$ after CLP. Defect in IL-1 signaling phenocopied PKR deficiency or inhibition in CLP-induced bacterial sepsis. Taken together, these findings identified a critical role of the PKR signaling pathway in antibacterial immunity.

(c) 2020 The Author(s)

Published by S. Karger AG, Base

karger@karger.com www.karger.com/jin

Karger"

BOPEN ACCESS
(C) 2020 The Author(s)

Published by S. Karger AG, Basel

This article is licensed under the Creative Commons AttributionNonCommercial-NoDerivatives 4.0 International License (CC BYNC-ND) (http://www.karger.com/Services/OpenAccessLicense) Usage and distribution for commercial purposes as well as any distribution of modified material requires written permission.

\section{Introduction}

Double-stranded RNA dependent kinase R (PKR) is an interferon (IFN)-inducible serine/threonine kinase, which contains 2 RNA-binding domains at the C-terminal and a kinase domain at the N-terminal [1-4]. Upon binding to the double-stranded (ds) RNA molecules, PKR uncovers its intramolecular dimerization domain, culminating in PKR dimerization, autophosphorylation, and activation [2-4]. As dsRNA is a general by-product of viral infection, PKR was originally identified as an antiviral molecule [3-5]. PKR contributes to the antiviral defense at multiple levels: (1) activated PKR recruits and phosphorylates eIF2a, resulting in inhibition of general protein synthesis; (2) PKR enhances the production of type 1 IFNs through the interaction with IFN response factor 3 (IRF3) pathways; and (3) activated PKR regulates actin dynamics and abrogates viral entry into cells by inhibiting gelsolin, a key actin-modifying protein [4-6]. We and others found that PKR promotes pyroptosis through physical interaction with inflammasome components

Y.Y. and L.X. are co-first authors.

Yiting Tang

Department of Physiology, School of Basic Medical Science, Central South University 172 Tonglupo Road

Changsha 410000 (China)

yitingtang@csu.edu.cn 
and enhances apoptosis [1,7-9]. Notably, both apoptosis and pyroptosis are important strategies for the host to directly eliminate the intracellular virus $[10,11]$. Owing to its important antiviral roles, most viruses have devised mechanisms to subvert PKR functions [3-5].

Although huge progress has been made in understanding how PKR responses to viral infections, little is known about the roles of PKR in antibacterial defenses. During the case of studying how PKR regulates inflammasome activation in response to various pathogen-associated molecular patterns (PAMPs), we unexpectedly found that Escherichia coli, which are abundant Gram-negative bacteria in the gastrointestinal tract, could induce robust PKR autophosphorylation [1]. This observation prompted us to investigate whether PKR plays a role in antibacterial defenses, especially in the context of intestinal bacterial infection. In current study, we found that genetic deletion of PKR or pharmacological inhibition of its kinase activity markedly increased bacterial loads, organ injury, and mortality in intestinal polymicrobial infection. PKR deficiency or inhibition significantly decreased the release of interleukin (IL)-1 $\beta$. Defect in IL-1 signaling phenocopied PKR deficiency or inhibition in intestinal polymicrobial infection. Thus, the PKR signaling pathway is critical for intestinal antibacterial defense.

\section{Materials and Methods}

\section{Mice}

The PKR-deficient mice and their wild-type (WT) littermate controls are in the C57BL/6 background as described previously [12]. All mice were bred in the animal facilities of Central South University. Experimental protocols were approved by the Institutional Animal Care and Use Committees of Central South University.

\section{Reagents}

The PKR inhibitor C16 was obtained from Abcam. Meropenem was obtained from Shenzhen Haibin Pharmaceutical Co., Ltd.

\section{CLP Procedure}

Experimental sepsis was induced by cecal ligation and puncture (CLP). Male or female mice that were 25-30 g in weight were used. The skin was disinfected with a $2 \%$ iodine tincture. Laparotomy was performed under 2\% isoflurane (Piramal Critical Care) with oxygen. To cause mortality ranged from 10 to $30 \%$ after CLP, $25 \%$ of the cecum was ligated and punctured twice with a 20-gauge needle. Saline $(1 \mathrm{~mL})$ was given subcutaneously for resuscitation immediately after operation. Mice were monitored daily by signs of a moribund state for lethality. For detection of TNF- $\alpha$ and IL- 6 serum level and gene expression in the intestine, mice were sacrificed at $4 \mathrm{~h}$ after CLP. For detection of IL-1 $\beta$, IL-1 $\alpha$, alanine aminotransferase (ALT), and aspartate transaminase (AST), mice were sacrificed at $18 \mathrm{~h}$ after CLP. Lung specimens were stained with $\mathrm{H} \& \mathrm{E}$. C16 was administered intraperitoneally $(500 \mu \mathrm{g} / \mathrm{kg})$ $1 \mathrm{~h}$ prior to CLP. Meropenem at the dose of $50 \mu \mathrm{g} / \mathrm{kg}$ was administered immediately after CLP and twice a day. The same vehicle was given to the sham operated mice.

\section{E. coli Injection}

Approximately $10^{8} \mathrm{CFU}$ of $E$. coli dissolved in $100-\mu \mathrm{L}$ phosphate-buffered solution (PBS) were injected intravenously into mice. Control mice underwent an injection with the same volume of PBS. After injection, animals were allowed to access to food and water freely and were observed for mortality or conducted examinations at predetermined time intervals.

\section{Macrophages Depletion}

WT and PKR-deficient mice were injected intraperitoneally with the first dose at $400 \mu \mathrm{L}$ of liposome-encapsulated clodronate or control lipsome (N.van Rooijen) $48 \mathrm{~h}$ before CLP and then were injected intravenously with the second dose at $250 \mu \mathrm{L}$ of liposomeencapsulated clodronate or control lipsome $24 \mathrm{~h}$ before CLP.

\section{Bacterial Counts}

Bacterial loads were measured in the lung, the liver, the blood, and the spleen. The homogenates of these organs or the blood were gradiently diluted in sterile saline and incubated at $37^{\circ} \mathrm{C}$ for $16 \mathrm{~h}$ on Luria-Bertani agar plates. Colony-forming units (CFU/mL.g) were counted as bacterial loads.

\section{ELISA Assay}

IL-1 $\beta$, IL-1 $\alpha$, IL-6, and TNF- $\alpha$ levels of serum samples were determined using ELISA kits as described in the protocol of the manufacturer (eBioscience).
Fig. 1. PKR activity is critical for antibacterial defense. a Kaplan Meier survival curves for the indicated genotypes of mice subjected to either mild CLP or sham operation. b The AST and ALT levels from the mice of indicated genotypes subjected to either mild CLP or sham operation. Serum samples were collected $18 \mathrm{~h}$ after CLP operation. c Histology of the lungs from the mice of indicated genotypes $(n=6)$ subjected to either mild CLP or sham operation. The lungs were collected $18 \mathrm{~h}$ after CLP operation and then stained with H\&E. d Kaplan Meier survival curves for the mice subjected to the sham operation or the mice subjected to mild CLP with intraperitoneal C16 $(500 \mu \mathrm{g} / \mathrm{kg})$ or DMSO injection. e The AST and ALT levels from the mice subjected to the sham operation or the mice subjected to mild CLP with intraperitoneal C16 $(500 \mu \mathrm{g} / \mathrm{kg})$ or DMSO injection. Serum samples were collected $18 \mathrm{~h}$ after CLP operation. $\mathbf{f}$ Histology of the lungs from the mice $(n=6)$ subjected to the sham operation or the mice subjected to mild CLP with intraperitoneal C16 $(500 \mu \mathrm{g} / \mathrm{kg})$ or DMSO injection. The lungs were collected $18 \mathrm{~h}$ after the operations and then stained with H\&E. Dots represent individual mice. ${ }^{*} p<0.05$; ${ }^{* *} p<0.01 ;{ }^{* * *} p<0.001 ;$ ns, not significant (Student's $t$ test and logrank test for survival). PKA, double-stranded RNA dependent kinase R; CLP, cecal ligation and puncture; AST, serum aspartate transaminase; ALT, alanine aminotransferase; WT, wild type.

(For figure see next page.) 


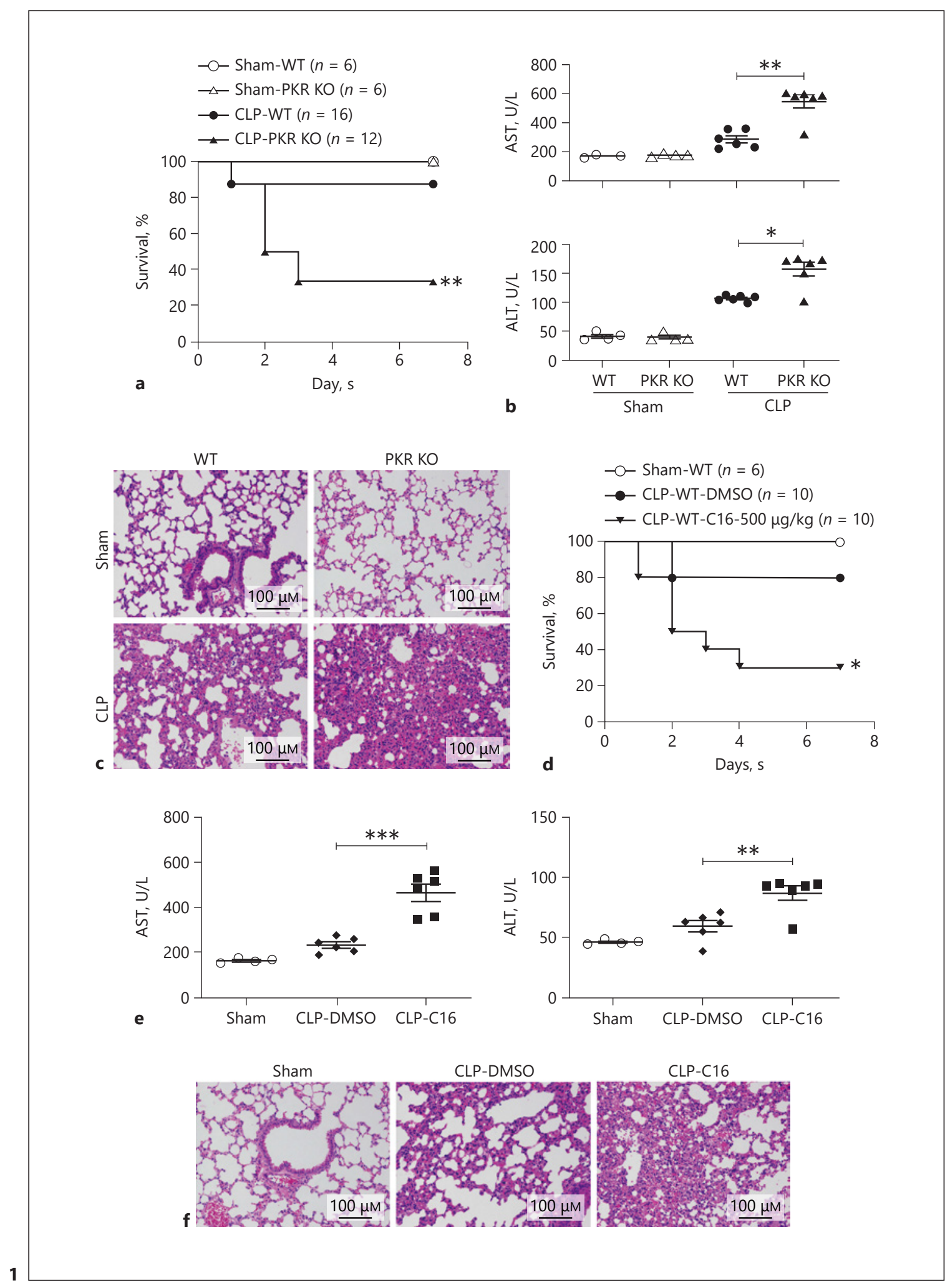

Yang/Xie/Zhong/Zhong/Meng/Xue/ Liang/Zhao/Tang 


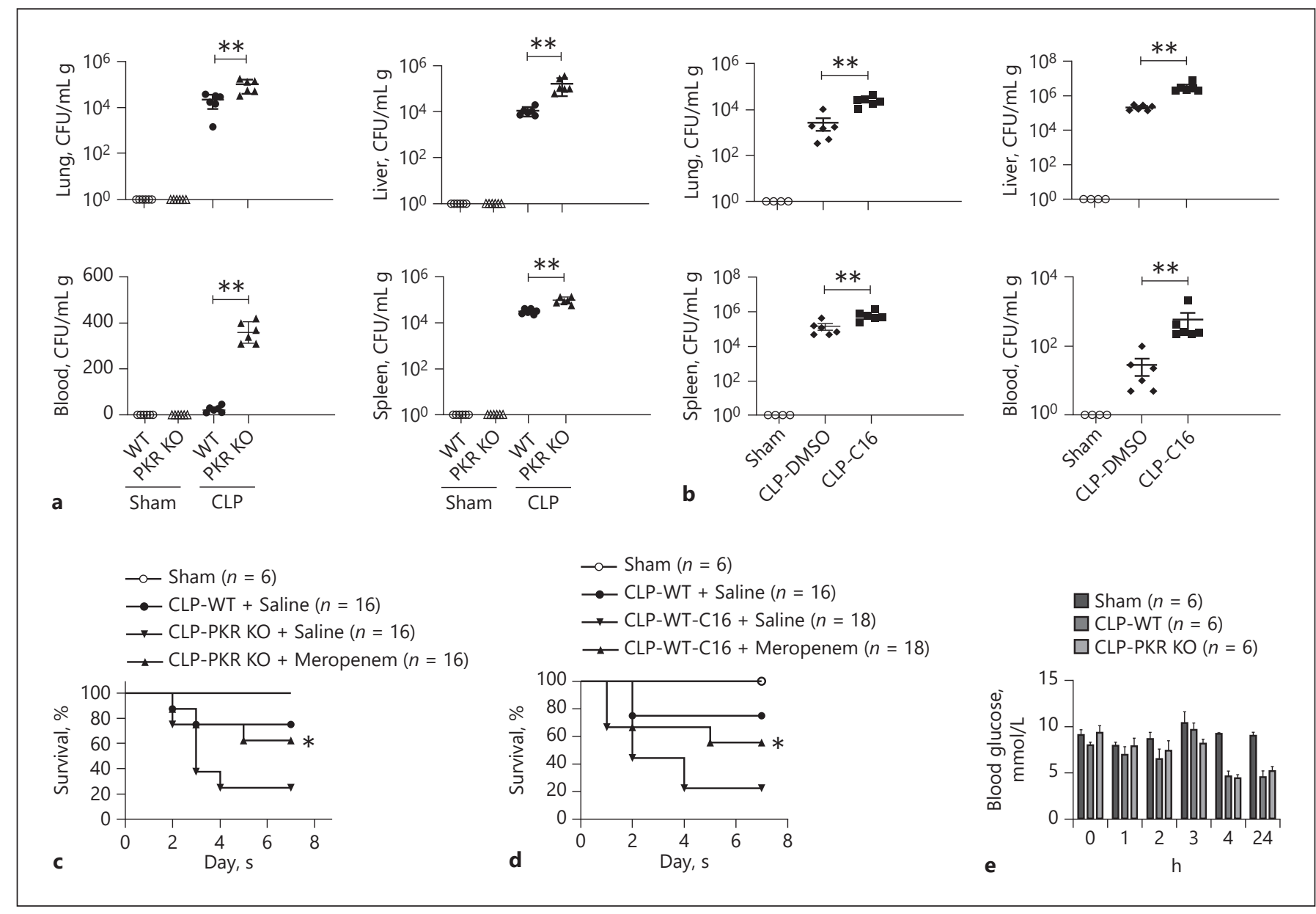

Fig. 2. PKR deficiency leads to higher bacterial loads during bacterial sepsis. a Bacterial load levels in the lung, liver, blood, and spleen from the mice of indicated genotypes subjected to either mild CLP or sham operation. The lung, liver, blood, and spleen were collected $18 \mathrm{~h}$ after the operations. b Bacterial load levels in the lung, liver, blood, and spleen from the mice subjected to the sham operation or the mice subjected to mild CLP with intraperitoneal C16 (500 $\mu \mathrm{g} / \mathrm{kg})$ or DMSO injection. The lung, liver, blood, and spleen were collected $18 \mathrm{~h}$ after the operations. c Kaplan Meier survival curves for the indicated genotype mice subjected to the sham operation or the mice subjected to mild CLP with or without

Fig. 3. PKR is dispensable for bacterial clearance after intravenous injection of E. coli. a Bacterial loads in the lung, liver, blood, and spleen from the mice of indicated genotypes after receiving intravenous injection of $E$. coli $\left(10^{8} \mathrm{CFU}\right)$ or PBS. The lung, liver, blood, and spleen were collected $18 \mathrm{~h}$ after the injections. b Bacterial loads in the lung, liver, blood, and spleen from the mice were intravenously injected with E. coli $\left(10^{8} \mathrm{CFU}\right)$ or PBS and simultaneously intraperitoneal Meropenem injection $(50 \mu \mathrm{g} / \mathrm{kg})$. d Kaplan Meier survival curves for the mice subjected to the sham operation or the mice subjected to mild CLP with or without intraperitoneal C16 injection $(500 \mu \mathrm{g} / \mathrm{kg})$ or intraperitoneal Meropenem injection (50 $\mu \mathrm{g} / \mathrm{kg}$ ). e Blood glucose levels for the indicated genotype mice subjected to either mild CLP or sham operation. Dots represent individual mice. ${ }^{*} p<0.05 ;{ }^{* *} p<0.01 ;{ }^{* * *} p<0.001$; ns, not significant (Student's $t$ test and log-rank test for survival). PKA, doublestranded RNA dependent kinase R; CLP, cecal ligation and puncture; WT, wild type.

intraperitoneally injected with $\mathrm{C} 16(500 \mu \mathrm{g} / \mathrm{kg})$ injection or DMSO. The lung, liver, blood, and spleen were collected $18 \mathrm{~h}$ after the injections. Dots represent individual mice. ${ }^{*} p<0.05$; ${ }^{* *} p<0.01$; $* * * p<0.001$; ns, not significant (Student's $t$ test). PKA, doublestranded RNA dependent kinase R; WT, wild type; PBS, phosphate-buffered solution.

(For figure see next page.) 


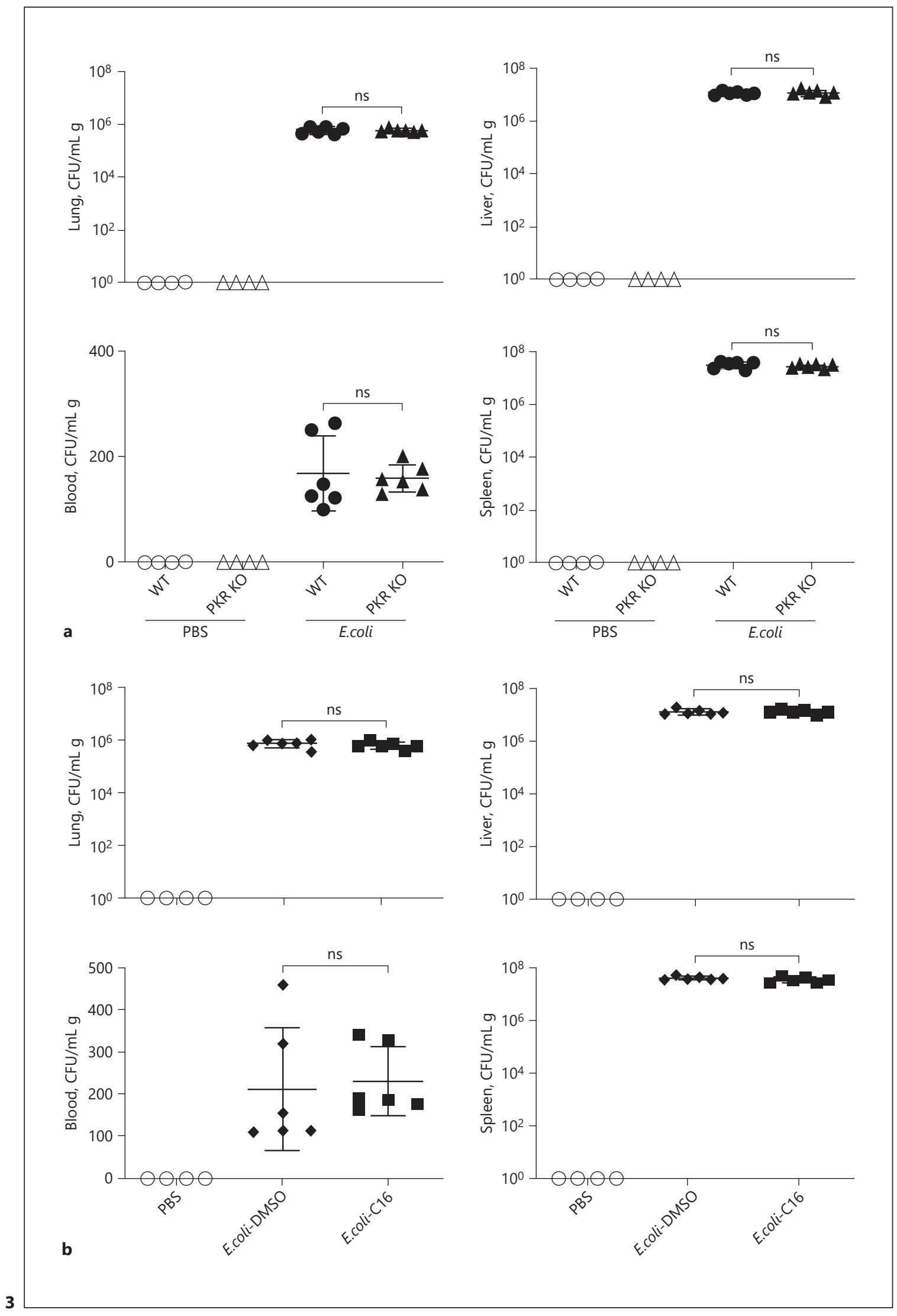


Fig. 4. $P K R$ is dispensable for bacterial clearance after intravenous injection of $E$. coli. a Kaplan Meier survival curves for the indicated genotype mice after receiving intravenous injection of E. coli $\left(10^{8} \mathrm{CFU}\right)$ or PBS. $\mathbf{b}$ The AST and ALT levels from mice of indicated genotypes after receiving intravenous injection of $E$. coli $\left(10^{8} \mathrm{CFU}\right)$ or PBS. Serum samples were collected $18 \mathrm{~h}$ after the injection. Dots represent individual mice. ${ }^{*} p<0.05 ;{ }^{* *} p<0.01 ;{ }^{* * *} p<0.001 ; \mathrm{ns}$, not significant (Student's $t$ test and logrank test for survival). PKA, double-stranded RNA dependent kinase R; AST, serum aspartate transaminase; ALT, alanine aminotransferase; WT, wild type; PBS, phosphate-buffered solution.

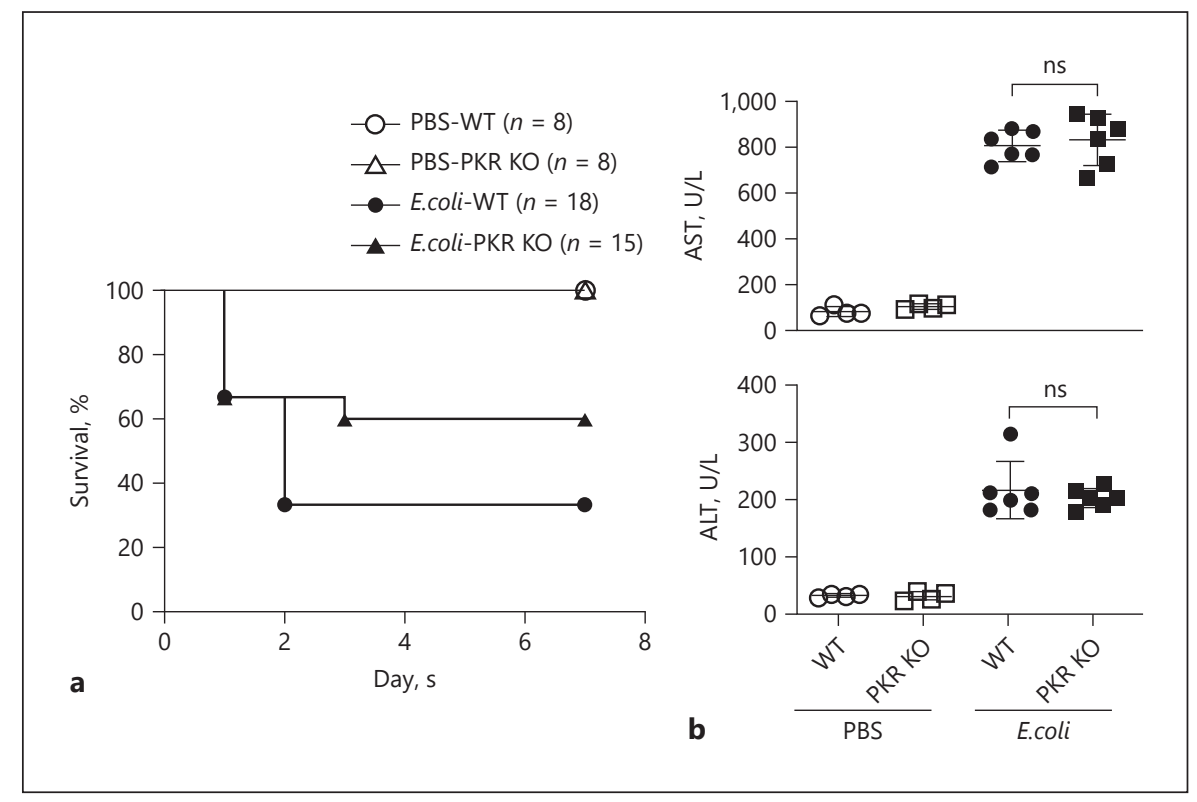

\section{$A L T$ and AST}

Serum samples were collected from various types of mice, and the levels of ALT and AST in serum were measured by Automatic Biochemical Analyzer (Chemray240).

\section{$q R T-P C R$}

Small/large intestine tissues are collected to determine the gene expression of TNF- $\alpha$ and IL- 6 at $4 \mathrm{~h}$ after CLP from WT and PKRdeficient mice. Total RNA was prepared using Trizol (Ambion, Waltham, MA, USA) according to the manufacturer's instructions, and the cDNA was synthesized from $3 \mu \mathrm{g}$ of total RNA using reverse transcriptase (TransGen Biotech, Beijing, China). Realtime PCR was performed using SYBR Green Master Mix (Vazyme Biotech Co., Ltd., Nanjing, China) and a C1000 Touch thermal cycler (Roche). The detector was programed with the following PCR conditions: 40 cycles for $5 \mathrm{~s}$ denaturation at $95^{\circ} \mathrm{C}$ and $30 \mathrm{~s}$ amplification at $60^{\circ} \mathrm{C}$. The mRNA level of target genes was normalized to that of GAPDH.

\section{IL-6_2_F 5'-CCAAGAGGTGAGTGCTTCCC-3' \\ IL-6_2_R 5'-CTGTTGTTCAGACTCTCTCCCT-3' TNF-a_F 5'-CCCTCACACTCAGATCATCTTCT-3' TNF-a_R 5'-GCTACGACGTGGGCTACAG-3' GAPDH_F $5^{\prime}$-TGGATTTGGACGCATTGGTC-3' GAPDH_R 5'-TTTGCACTGGTACGTGTTGAT-3'}

\section{Isolation of Bone Marrow Neutrophils}

Murine PMN from WT and PKR-deficient mice were isolated essentially as described [13]. Marrow cavities of the tibias and femurs of 8- to 12-week-old donor mice were flushed with DMEM $10 \%$ FCS media. After RBC hypotonic lysis, neutrophils were isolated by centrifugation over discontinuous Percoll (sigma, P4937) gradients at $500 \mathrm{~g}$ for $30 \mathrm{~min}$ at $28^{\circ} \mathrm{C}$, consisting of $55 /(\mathrm{vol} / \mathrm{vol}), 62$ (vol/vol), and $81 \%$ (vol/vol) Percoll in PBS, and then neutrophils were harvested from the $62 / 81 \%$ interface. At the end of the preparation, PMN were suspended in ice-cold HBSS containing $0.5 \mathrm{mM}$
$\mathrm{CaCl}_{2}, 1 \mathrm{mM} \mathrm{MgCl}$, and $5 \mathrm{mmD}$-glucose (HBSS) and kept in ice until use.

\section{Isolation of Peritoneal Macrophages}

Mice in age between 7 and 12 weeks received intraperitoneal injection with $3 \mathrm{~mL}$ of sterile $3 \%$ thioglycollate broth to induce peritoneal macrophages. Cells were harvested after $72 \mathrm{~h}$ by peritoneal lavage with $5 \mathrm{~mL}$ of RPMI medium 1,640 (Gibco) and passed through a $40-\mu \mathrm{m}$ cell strainer. After centrifugation $(800 \mathrm{rpm}, 5$ min), the cells were resuspended in RPMI medium 1,640 containing $10 \%$ fetal bovine serum (Gibco) and $1 \%$ antibiotics (Gibco) and plated in a 24-well plate overnight.

In vitro Murine Neutrophil and Macrophage Infection Assays

Neutrophils and macrophages from WT and PKR-deficient mice were seeded at a density of $1 \times 10^{7} / \mathrm{mL}$ and $1 \times 10^{6} / \mathrm{mL}$ in RPMI 1640 supplemented with $10 \%$ fetal calf serum and then infected with $E$. coli at a multiplicity of infection (MOI) of 10 . The cells were then incubated at $37^{\circ} \mathrm{C}$ for $2 \mathrm{~h}$ to allow bacterial uptake, before adding gentamicin $(100 \mathrm{~g} / \mathrm{mL}$; Life Technologies $)$ to the culture medium for the remainder of the assay to kill extracellular bacteria. The cells were then further incubated for $16 \mathrm{~h}$ before harvesting cell-free supernatants for cytokine assays.

\section{Statistical Analysis}

All data were analyzed using GraphPad Prism software (version 5.01). Data were analyzed using two-tailed Student's $t$ test for the comparison between 2 groups. Data were analyzed using oneway ANOVA followed by the post hoc Bonferroni test for comparison between multiple groups. Survival data were analyzed using the log-rank test. A $p$ value $<0.05$ was considered as statistically significant for all experiments. Dots represent individual mice in both vivo experiments and vitro experiments, graphs show the mean \pm SD of technical replicates and are representative of three independent experiments. 


\section{Result}

\section{PKR Activity Is Critical for Antibacterial Defense}

To test whether PKR activation contributes to the antibacterial defenses during bacterial infection, we subjected PKR-deficient mice, in which the kinase domain of PKR was deleted $[12,14]$, and their WT littermates to mild degree of CLP. Most of the WT mice survived after CLP, whereas most PKR-deficient mice succumbed to the CLP-induced polymicrobial infection (Fig. 1a). Accordingly, PKR-deficient mice had significantly higher levels of serum AST and ALT in CLP, as compared to their WT controls (Fig. 1b). Further, PKR deficiency significantly increased pulmonary leukocyte infiltration after CLP (Fig. 1c). To test whether pharmacological inhibition of PKR could impair the antibacterial defenses in bacterial sepsis, we pretreated the mice with either C16, a specific PKR inhibitor [15], or vehicle. Notably, pharmacological inhibition of PKR significantly enhanced liver and lung injury and promoted lethality after CLP (Fig. 1d-f). Taken together, these findings suggest that PKR activity is critical for antibacterial defense.

\section{PKR Deficiency Leads to Higher Bacterial Loads}

during Bacterial Sepsis

To further confirm that PKR activation is important for antibacterial defenses in sepsis, we measured the bacterial loads in the lungs, livers, blood, and spleens of PKRdeficient mice and their WT littermate controls. Deletion of the PKR kinase domain led to significantly higher bacterial loads in the lung, liver, blood, and spleen (Fig. 2a). Accordingly, pharmacological inhibition of PKR markedly increased the bacterial loads in these organs after CLP (Fig. 2b). Next we determined whether the increased bacterial loads resulted from PKR deletion or inhibition promote organ injury and lethality after CLP. PKR-deficient mice were pretreated with broad-spectrum antibiotics or placebo before CLP. Administration of antibiotics rescued PKR-deficient mice from lethal sepsis (Fig. 2c). Similar observations were made in C16-treated mice (Fig. 2d). Maintaining blood glucose at certain level is essential for the host to survive bacterial infection. Thus, we next measured blood glucose levels of PKR-deficient mice and their WT controls after CLP. We observed that PKR-deficient mice and their WT controls had comparable levels of blood glucose after CLP (Fig. 2e). Together, PKR deficiency or inhibition impairs antibacterial defenses in bacterial sepsis.
PKR Is Dispensable for Bacterial Clearance after

Intravenous Injection of E. coli

Next we determined whether the increased bacterial loads in PKR-deficient mice after CLP were due to the impaired capacity of bacterial clearance or the dissemination of enteric bacteria. To test this, PKR-deficient mice and their WT littermate controls were intravenously injected with the same amount of E. coli, an abundant microbe in the gastrointestinal tract. However, we observed that PKR-deficient mice and their WT controls had comparable bacterial loads in the lung, liver, blood, and spleen (Fig. 3a). Similar observations were made in mice treated with $\mathrm{C} 16$ or vehicle (Fig. 3b). PKR deficiency failed to promote lethality after intravenous injection of $E$. coli (Fig. 4a). Accordingly, PKR-deficient mice and their WT controls had comparable amount of serum levels of AST and ALT (Fig. 4b). Together with other data (Fig. 2), these findings suggested that the increased bacterial loads in PKR-deficient mice during intestinal polymicrobial infection might be due to impaired dissemination of enteric bacteria, rather than impaired capacity of bacterial clearance.

\section{PKR Deficiency Leads to Impaired Production of IL-1} in Bacterial Sepsis

We and others previously found that PKR broadly regulate the activation of inflammasome, an intracellular protein complex that mediates the enzymatic cleavage of caspase-1 [1,7-9]. This process leads to the maturation and release of IL-1 $\beta$, which critically regulates the antibacterial defenses [16]. Thus, we next measured serum levels of IL- $1 \beta$ and IL- $1 \alpha$ using CLP models. Genetic deletion of the PKR kinase domain significantly inhibited the release of IL- $1 \beta$ and IL- $1 \alpha$ after CLP (Fig. 5a). Accordingly, pharmacological inhibition of PKR by $\mathrm{C} 16$ also led to markedly decreased serum levels of IL- $1 \beta$ and IL- $1 \alpha$ (Fig. 5b). However, PKR deficiency did not affect the serum levels (Fig. 5c) of IL-6 and tumor necrosis factor (TNF), as well as the gene expression (Fig. 5d) of IL-6 and TNF in the intestine after CLP. Further, genetic deletion of the PKR kinase domain failed to affect the bacterial loads in the intestine (Fig. 5e). Together, these data demonstrated that PKR activity is critical for the release of IL-1 in experimental bacterial sepsis.

\section{Defect in IL-1 Signaling Phenocopied PKR Deficiency in Bacterial Sepsis}

Next we determined whether the impaired IL-1 signaling phenocopied PKR deficiency in bacterial sepsis. IL-1R $\mathrm{KO}$ mice and their WT controls were subjected to mild
Yang/Xie/Zhong/Zhong/Meng/Xue/ Liang/Zhao/Tang 
Fig. 5. PKR deficiency leads to impaired production of IL-1 $\beta$ in bacterial sepsis. a Serum IL-1 $\beta$ and IL-1 $\alpha$ levels from the mice of indicated genotypes subjected to either mild CLP or sham operation. Serum samples were collected $18 \mathrm{~h}$ after the operations. b Serum IL- $1 \beta$ and IL- $1 \alpha$ levels from mice subjected to either mild CLP or sham operation after using the C16 inhibitor. Serum samples were collected $18 \mathrm{~h}$ after the operations. c Serum levels of IL-6 and TNF from the mice of indicated genotypes subjected to either mild CLP or sham operation. Serum samples were collected $4 \mathrm{~h}$ after the operations. $\mathbf{d}$ Gene expression in the intestine of IL- 6 and TNF from the mice of indicated genotypes subjected to either mild CLP or sham operation. Intestine tissues were collected $4 \mathrm{~h}$ after the operations. e Bacterial load levels in the intestine from the mice of indicated genotypes subjected to either mild CLP or sham operation. Dots represent individual mice. ${ }^{*} p<0.05 ;{ }^{* *} p<0.01 ;{ }^{* * *} p<0.001$; ns, not significant (Student's $t$ test). PKA, doublestranded RNA dependent kinase R; IL, interleukin; CLP, cecal ligation and puncture; WT, wild type.
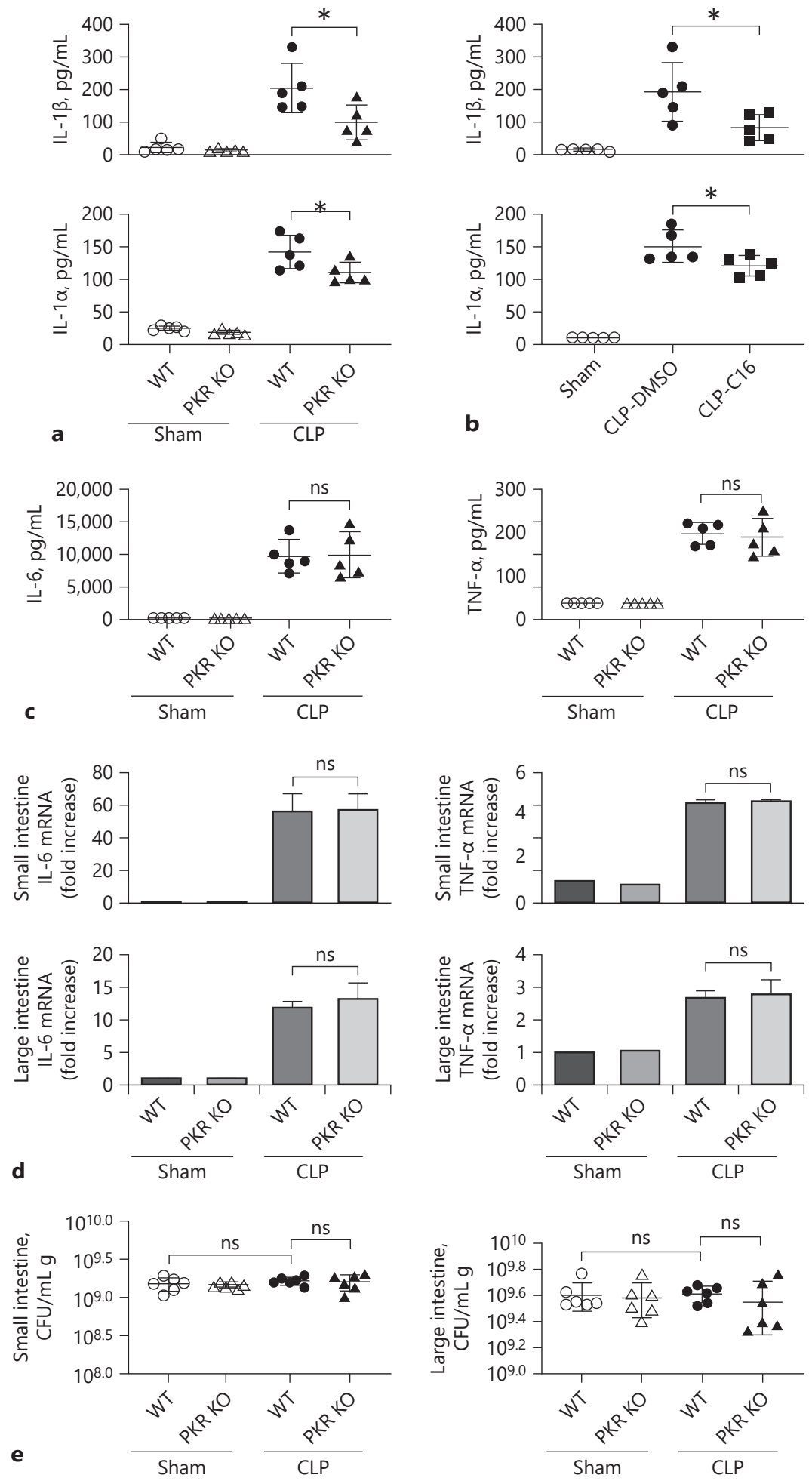


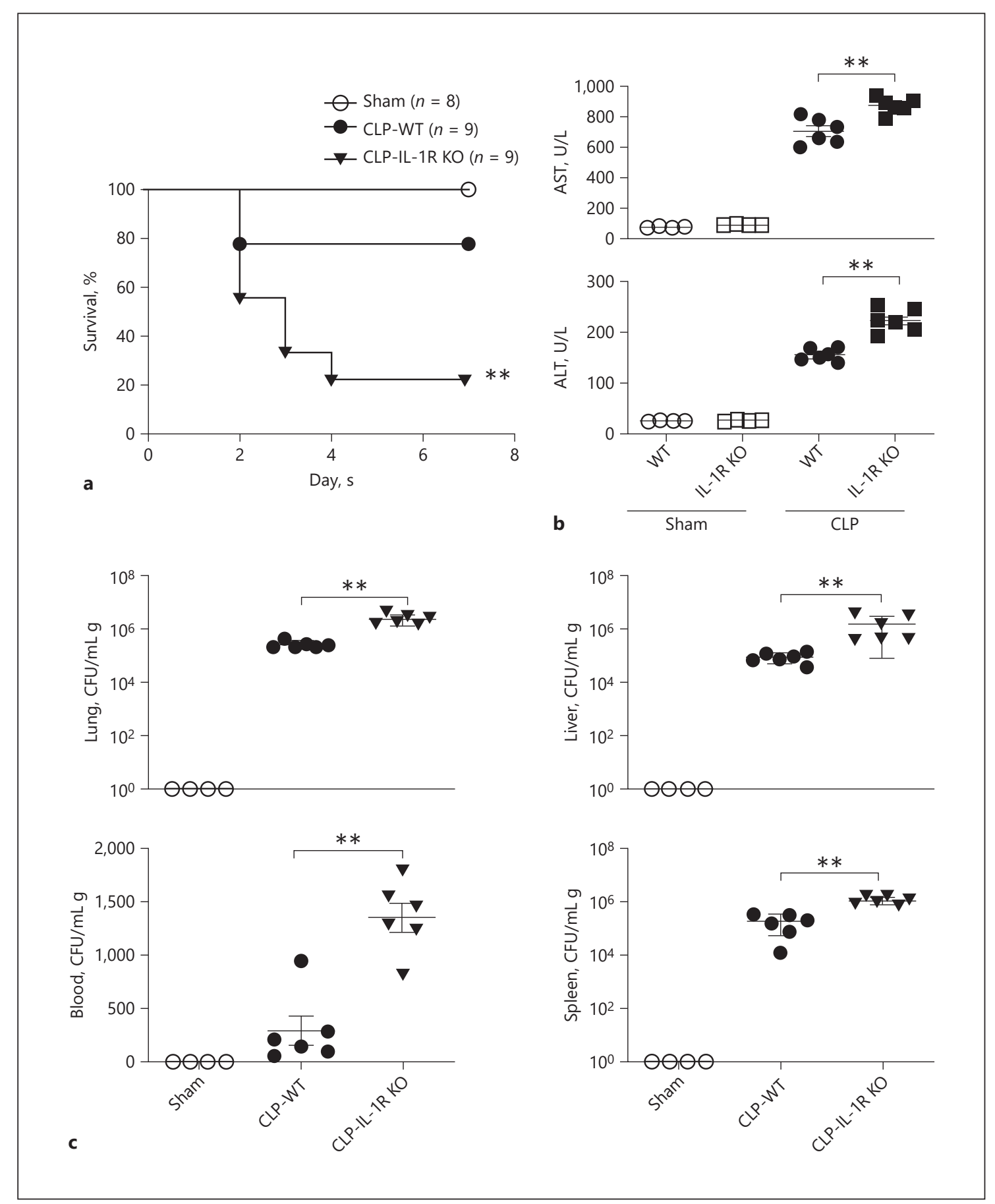

Fig. 6. Defect in IL-1 signaling phenocopied PKR deficiency in bacterial sepsis. a Kaplan Meier survival curves for the indicated genotype mice subjected to either mild CLP or sham operation. b The AST and ALT levels from the mice of indicated genotypes subjected to either mild CLP or sham operation. Serum samples were collected $18 \mathrm{~h}$ after the operations. c Bacterial load levels in the lung, liver, blood, and spleen from the mice of indicated geno- types subjected to either mild CLP or sham operation. The lung, liver, blood, and spleen were collected $18 \mathrm{~h}$ after the operations. Dots represent individual mice. ${ }^{*} p<0.05 ;{ }^{* *} p<0.01 ;{ }^{* * *} p<0.001$; ns, not significant (Student's $t$ test and log-rank test for survival). PKA, double-stranded RNA dependent kinase R; CLP, cecal ligation and puncture; AST, serum aspartate transaminase; ALT, alanine aminotransferase; WT, wild type. 


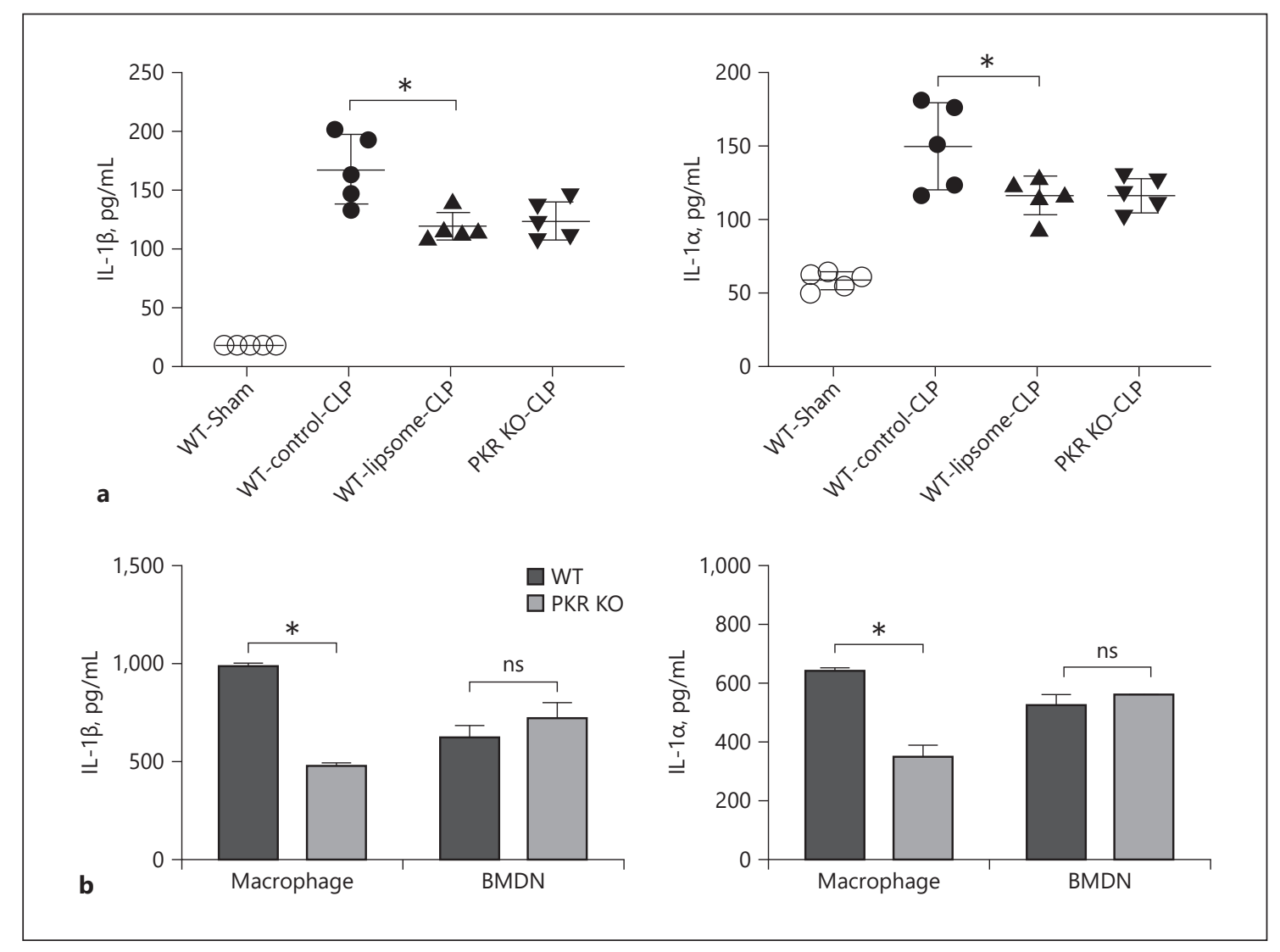

Fig. 7. $\mathrm{PKR}$ in macrophages is important for IL-1 secretion. a Serum IL- $1 \beta$ and IL- $1 \alpha$ levels from the mice with or without macrophage depletion subjected to either mild CLP or sham operation. Serum samples were collected $18 \mathrm{~h}$ after the operations. Dots represent individual mice. ${ }^{*} p<0.05 ;{ }^{* *} p<0.01 ;{ }^{* * *} p<0.001$; ns, not significant (Student's $t$ test). b ELISA for IL- $1 \beta$ and IL- $1 \alpha$ in the supernatants of neutrophils or macrophages from WT and PKRdeficient mice infected with E. coli at an MOI of 10 for $2 \mathrm{~h}$ uptake

CLP, in which most of the WT mice were survived, and deletion of IL-1R significantly promoted CLP-induced lethality (Fig. 6a). IL-1R KO mice displayed significantly higher serum levels of AST and ALT in CLP, as compared to those of their WT controls (Fig. 6b). Further, defect in IL-1 signaling was associated with significantly increased bacterial loads in the lung, liver, blood, and spleen (Fig. 6c). Collectively, defect in IL-1 signaling phenocopied PKR deficiency during intestinal polymicrobial infection.

\section{PKR in Macrophages Is Important for IL-1 Secretion}

We and others previously show that macrophages constitutively express PKR [17] and that PKR is critical for IL-1 secretion during sepsis [1]. To determine wheth- and then killed by gentamicin $(100 \mathrm{~g} / \mathrm{mL})$. Cell-free supernatants were collected after $16 \mathrm{~h}$ further incubation. Graphs show the mean \pm SD of technical replicates and are representative of 3 independent experiments. ${ }^{*} p<0.05 ;{ }^{* *} p<0.01$; ${ }^{* *} p<0.001$; ns, not significant (Student's $t$ test). PKA, double-stranded RNA dependent kinase R; IL, interleukin; CLP, cecal ligation and puncture; WT, wild type; MOI, multiplicity of infection.

er PKR-dependent IL-1 secretion mainly occurs in macrophages during sepsis, we depleted macrophages in vivo by using liposomes-clodronate. Administration of liposomes-clodronate markedly decreased IL- $1 \beta$ and IL- $1 \alpha$ secretion and significantly increased the bacterial loads after CLP (Fig. 7a). To further support the notion that macrophage PKR is important for the IL-1 secretion in bacterial sepsis, we stimulated mouse macrophages or neutrophils isolated from WT or PKR-deficient mice with E. coli. Deletion of the PKR kinase domain significantly reduced IL- $1 \beta$ and IL- $1 \alpha$ secretion in macrophages but not neutrophils (Fig. 7b). Collectively, these findings clearly suggest that PKR in macrophages is important for IL-1 secretion. 


\section{Discussion}

In this study, we showed for the first time that PKR is an important regulator of intestinal antibacterial immunity. The antiviral function of PKR was discovered more than 30 years ago [18]. The best-known model of how PKR combats viral infection is that autophosphorylated PKR recruits and phosphorylates its substrate eIF2a upon recognizing viral double-stranded RNA, resulting in inhibition of general protein synthesis [2-4]. Though PKR is originally identified as an intracellular viral RNA sensor, bacterial endotoxin (lipopolysaccharide [LPS]), the major cell-wall component of Gramnegative bacteria, is also able to induce PKR phosphorylation and activation through toll-like receptor (TLR) 4 and its adapter molecule TIR-domain-containing adapter-inducing interferon- $\beta$ (TRIF). In this scenario, LPS-induced PKR phosphorylation is essential for bacteria-induced macrophage death [17]. As macrophages are important innate immune cells, this study implicates that PKR might regulate antibacterial immunity. However, the roles of PKR in antibacterial defenses at the organism level are yet to be found. Here we showed that PKR activity is critical for the antibacterial defense during experimental sepsis.

In this context, PKR regulates the production of IL- $1 \beta$, which plays important roles in preventing the dissemination of enteric bacteria after CLP through neutrophil recruitment. Intriguingly, PKR deficiency failed to affect the bacterial clearance and the mortality when E. coli were directly injected into the veins, suggesting that PKR is important for the antibacterial defenses in the peritoneal cavity but dispensable for bacterial clearance during bacteremia. By using E. coli-induced peritonitis model, we previously found that PKR deficiency significantly inhibits neutrophil recruitment in the peritoneal cavity [1]. Macrophages are abundant in the peritoneal cavity. It is noteworthy that PKR in macrophages is important for IL-1 secretion, which leads to subsequent neutrophil recruitment. As neutrophil influx is critical for bacterial clearance in tissues, our data suggest an important role of peritoneal macrophage PKR in the antibacterial defenses during bacterial peritonitis. In this context, Gram-negative bacteria induce PKR activation in peritoneal macrophages through TLR4, leading to IL-1 secretion and subsequent neutrophil recruitment, which prevents the dissemination of bacteria.

The maturation and release of IL- $1 \beta$ is mediated by caspase-1, which is activated by intracellular protein complexes, termed inflammasomes $[1,7]$. The well-char- acterized inflammasomes include NLRP1, NLRP3, AIM2, and NLRC4, all of which contribute to the antibacterial immunity [19-22]. However, different types of inflammasomes response to distinct stimuli during bacterial infection [19-22]. For examples, NLRP1 is activated by anthrax lethal toxin or MDP. The AIM2 or NLRC4 inflammasome responds to intracellular bacterial DNA or flagellin, respectively. Further, Gram-negative bacteria activate the NLRP3 inflammasome through caspase-11 $[23,24]$. We and others previously showed that PKR regulates the activation of multiple types of inflammasomes [1, 7-9]. It is unlikely that the inflammasome components are the substrate of PKR [1]. However, the autophosphorylation of PKR could increase the affinity between PKR and inflammasomes, which is required for PKR to promote the activation of multiple types of inflammasomes $[1,7]$. One remaining unsolved question is as follows: which type of inflammasome is activated by PKR in intestinal polymicrobial infection? As the 4 types of inflammasomes are all expressed in the gastrointestinal tract [21, 25-27], it is possible that PKR might signal through multiple types of inflammasomes during intestinal polymicrobial infection. Taken together, our study identifies a critical role of the PKR-inflammasome pathway in the antibacterial immunity.

\section{Acknowledgement}

We thank Xiangyu Wang for technical assistance.

\section{Statement of Ethics}

Experimental protocols were approved by the Institutional Animal Care and Use Committees of Central South University.

\section{Disclosure Statement}

The authors have no conflicts of interest to declare.

\section{Funding Sources}

This study was supported by the National Natural Science Foundation of China (No. 81971893 [Y.T.] and No. 81700127 [F.L.]) and Innovation-Driven Scientific Project of CSU (B.L.).
Yang/Xie/Zhong/Zhong/Meng/Xue/ Liang/Zhao/Tang 


\section{Author Contributions}

Yiting Tang conceived the project, designed the experiments, supervised the study, and wrote the paper; Yanliang Yang and Lingli Xie designed and performed the experiments, analyzed the data and made the figures; Yanjun Zhong, Xiaoli Zhong, Ran Meng, and Qianqian Xue assisted in the in vivo experiments; Kai zhao and Fang liang assisted in data interpretation and edited the manuscript..

\section{References}

1 Lu B, Nakamura T, Inouye K, Li J, Tang Y, Lundbäck P, et al. Novel role of PKR in inflammasome activation and HMGB1 release. Nature. 2012;488(7413):670-4.

2 Dar AC, Dever TE, Sicheri F. Higher-order substrate recognition of eIF2alpha by the RNA-dependent protein kinase PKR. Cell. 2005; 122(6):887-900.

3 Dey M, Cao C, Dar AC, Tamura T, Ozato K, Sicheri F, et al. Mechanistic link between PKR dimerization, autophosphorylation, and eIF2alpha substrate recognition. Cell. 2005; 122(6):901-13.

4 Taylor SS, Haste NM, Ghosh G. PKR and eIF2alpha: integration of kinase dimerization, activation, and substrate docking. Cell. 2005; 122(6):823-5.

5 Tang Y, Zhao X, Antoine D, Xiao X, Wang H, Andersson U, et al. Regulation of posttranslational modifications of HMGB1 during immune responses. Antioxid Redox Signal. 2016;24(12):620-34.

6 Irving AT, Wang D, Vasilevski O, Latchoumanin O, Kozer N, Clayton AH, et al. Regulation of actin dynamics by protein kinase $\mathrm{R}$ control of gelsolin enforces basal innate immune defense. Immunity. 2012;36(5):795806.

7 Hett EC, Slater LH, Mark KG, Kawate T, Monks BG, Stutz A, et al. Chemical genetics reveals a kinase-independent role for protein kinase R in pyroptosis. Nat Chem Biol. 2013; 9(6):398-405

8 Boriushkin E, Wang JJ, Li J, Bhatta M, Zhang SX. p58(IPK) suppresses NLRP3 inflammasome activation and IL- $1 \beta$ production via inhibition of PKR in macrophages. Sci Rep. 2016;6:25013
9 Xie M, Yu Y, Kang R, Zhu S, Yang L, Zeng L, et al. PKM2-dependent glycolysis promotes NLRP3 and AIM2 inflammasome activation. Nat Commun. 2016;7:13280.

10 Rathinam VA, Jiang Z, Waggoner SN, Sharma S, Cole LE, Waggoner L, et al. The AIM2 inflammasome is essential for host defense against cytosolic bacteria and DNA viruses. Nat Immunol. 2010;11(5):395-402.

11 Chen IY, Ichinohe T. Response of host inflammasomes to viral infection. Trends $\mathrm{Mi}$ crobiol. 2015;23(1):55-63.

12 Nakamura T, Furuhashi M, Li P, Cao H, Tuncman G, Sonenberg N, et al. Doublestranded RNA-dependent protein kinase links pathogen sensing with stress and metabolic homeostasis. Cell. 2010;140(3):338-48.

13 Lowell CA, Fumagalli L, Berton G. Deficiency of Src family kinases p59/61hck and p58c-fgr results in defective adhesion-dependent neutrophil functions. J Cell Biol. 1996;133(4): 895-910.

14 Abraham N, Stojdl DF, Duncan PI, Méthot N, Ishii T, Dubé M, et al. Characterization of transgenic mice with targeted disruption of the catalytic domain of the double-stranded RNA-dependent protein kinase, PKR. J Biol Chem. 1999;274(9):5953-62.

15 Tronel C, Page G, Bodard S, Chalon S, Antier D. The specific PKR inhibitor C16 prevents apoptosis and IL- $1 \beta$ production in an acute excitotoxic rat model with a neuroinflammatory component. Neurochem Int. 2014;64: 73-83.

16 Krumm B, Xiang Y, Deng J. Structural biology of the IL-1 superfamily: key cytokines in the regulation of immune and inflammatory responses. Protein Sci. 2014;23(5):526-38.

17 Hsu LC, Park JM, Zhang K, Luo JL, Maeda S, Kaufman RJ, et al. The protein kinase PKR is required for macrophage apoptosis after activation of Toll-like receptor 4. Nature. 2004; 428(6980):341-5.
18 Edery I, Petryshyn R, Sonenberg N. Activation of double-stranded RNA-dependent kinase (dsl) by the TAR region of HIV-1 mRNA: a novel translational control mechanism. Cell. 1989;56(2):303-12.

19 Strowig T, Henao-Mejia J, Elinav E, Flavell R. Inflammasomes in health and disease. $\mathrm{Na}$ ture. 2012;481(7381):278-86.

20 Chavarría-Smith J, Vance RE. The NLRP1 inflammasomes. Immunol Rev. 2015;265(1): 22-34.

21 Rathinam VA, Fitzgerald KA. Inflammasome complexes: emerging mechanisms and effector functions. Cell. 2016;165(4):792-800.

22 Schroder K, Tschopp J. The inflammasomes. Cell. 2010;140(6):821-32.

23 Kayagaki N, Warming S, Lamkanfi M, Vande Walle L, Louie S, Dong J, et al. Non-canonical inflammasome activation targets caspase-11. Nature. 2011;479(7371):117-21.

24 Rathinam VA, Vanaja SK, Waggoner L, Sokolovska A, Becker C, Stuart LM, et al. TRIF licenses caspase-11-dependent NLRP3 inflammasome activation by gram-negative bacteria. Cell. 2012;150(3):606-19.

$25 \mathrm{Hu}$ B, Jin C, Li HB, Tong J, Ouyang X, Cetinbas NM, et al. The DNA-sensing AIM2 inflammasome controls radiation-induced cell death and tissue injury. Science. 2016; 354(6313):765-8.

26 Schieber AM, Lee YM, Chang MW, Leblanc M, Collins B, Downes M, et al. Disease tolerance mediated by microbiome E. coli involves inflammasome and IGF-1 signaling. Science. 2015;350(6260):558-63.

27 Allen IC, TeKippe EM, Woodford RM, Uronis JM, Holl EK, Rogers AB, et al. The NLRP3 inflammasome functions as a negative regulator of tumorigenesis during colitis-associated cancer. J Exp Med. 2010;207(5):1045-56. 